Pacific Journal of Mathematic 


\title{
NONCOMMUTATIVE RINGS WHOSE CYCLIC MODULES HAVE CYCLIC INJECTIVE HULLS
}

\author{
B. L. OSOFSKY
}

A ring $R$ is called hypercyclic if every cyclic $R$-module has cyclic injective hull. If $R$ is hypercyclic and $R / J$ artinian, then $R$ is a ring direct sum of matrix rings over local hypercyclic rings. The structure of local hypercyclic rings is studied.

In [1] and [2] Caldwell has characterized rings $R$ (with 1) such that

(i) $R$ is commutative, and

(ii) Every cyclic $R$-module has cyclic injective hull. In particular, every such ring has the property

(iii) $R / J$ is artinian, where $J$ is the Jacobson radical of $R$.

In $\S 1$, the commutativity condition (i) is dropped, and it is shown that rings satisfying (ii) and (iii) are direct sums of matrix rings over local rings satisfying (ii). In $\S 2$ local rings satisfying (ii) are studied. These are, with one possible exception, almost commutative in the sense that $x R=R x$ for all $x \in R$. For such rings, Caldwell's description in the commutative case goes through. The possible exception would imply the existence of a simple radical ring (without 1 of course) which is not nil and whose right ideals and left ideals are linearly ordered. This is why the word "possible" is inserted.

In this paper, $R$ will denote a ring with 1 , and all modules will be unital right $R$-modules. If $M_{R}$ is a module, $E\left(M_{R}\right)$ will denote the injective hull of $M . M_{R}$ is an essential extension of $N_{R}$ will be denoted $M^{\prime} \supseteqq N$ or $N \cong$. Following Caldwell's terminology in [1] and [2], $R$ will be called hypercyclic if $R$ satisfies (ii), that is, $E(R / I)$ is cyclic for every right ideal $I$. The socle of $M$ will be denoted $S(M)$, and $R_{n}$ will denote the ring of $n \times n$ matrices over $R . \quad R$ is called regular if every finitely generated right ideal is generated by an idempotent.

1. Hypercyclic rings with chain conditions on $R / J$. In this section we study hypercyclic rings such that $R / J$ is artinian. Such rings will be called restricted hypercyclic. We do not actually use the full force of $R / J$ artinian; it is equivalent to assume that $R$ is hypercyclic and $R / I$ has ascending chain condition on direct summands for all right ideals $I$. Thus if $R$ is hypercyclic, the ascending chain condition on $(R / J)_{R}$ will imply $R$ is restricted hypercyclic also. We start by quoting several known results. 
Lemma 1.1. Let $M$ be a finitely generated $R$-module. Then $M J=M \Leftrightarrow M=0$.

Proof. See Jacobson [6], Theorem 10. I suspect the name Nakayama usually associated with this lemma comes from [11], where Nakayama reformulates the statement of Jacobson's theorem by throwing out extraneous hypotheses.

Proposition 1.2. Let $R=I_{1} \oplus \cdots \oplus I_{n}$, where $\left\{I_{j} \mid 1 \leqq j \leqq n\right\}$ are isomorphic right ideals. Let $R^{\prime}=\operatorname{Hom}_{R}\left(I_{1}, I_{1}\right)$. Then $R \approx\left(R^{\prime}\right)_{n}$.

Proof. See Jacobson [8], p. 52.

Proposition 1.3. Let $R_{R}$ be injective. Then $R / J$ is regular, and for each set $\left\{\varepsilon_{i} \mid 1 \leqq i \leqq n\right\}$ of orthogonal idempotents in $R / J$ such that $\sum_{i=1}^{n} \varepsilon_{i}=1+J$, there exists $\left\{e_{i} \mid 1 \leqq i \leqq n\right\}$ orthogonal idempotents in $R$ with $e_{i}+J=\varepsilon_{i}$ and $\sum_{i=1}^{n} e_{i}=1$.

Proof. See Faith and Utumi [5].

Proposition 1.4. Let $e=e^{2}, f=f^{2} \in R$. Then $e R \approx f R \Leftrightarrow e R / e J \approx$ $f R / f J$.

Proof. See [8], p. 53.

Proposition 1.5. Let $F=\sum_{i=1}^{n} x_{i} R$ be a free $R$-module with free basis $\left\{x_{i} \mid 1 \leqq i \leqq n\right\}$. Then $M_{R} \rightarrow \operatorname{Hom}_{R}(F, M)$ is a category isomorphism between the category of right $R$-modules and the category of right $R_{n}$-modules with inverse $N_{R_{n}} \rightarrow N \otimes_{R_{n}} F$.

Proof. See Morita [10], Theorem 3.4.

Proposition 1.6. Let $R$ be restricted hypercyclic. Then $R$ '? $S(R)$.

Proof. See Caldwell [1], Theorem 3.5. $R / J=\sum_{i=1}^{n} \oplus S_{i}, \quad S_{i}$ simple, and every simple $R$-module is isomorphic to some $S_{i}$. Hence $E(R / J)=\sum \oplus E\left(S_{i}\right)$ is faithful, and $E\left(S_{i}\right)=x_{i} R$ for some $x_{i}$. Let $D_{i}=\left\{r \in R \mid x_{i} r \in S_{i}\right\}$. Then $D_{i} \subseteq^{\prime} R$ since $E\left(S_{i}\right)^{\prime} \supseteqq S_{i}$ and $\bigcap_{i=1}^{n} D_{i} \subseteq^{\prime} R$. Then $E(R / J) \cdot \bigcap_{i=1}^{n} D_{i} \subseteq R / J$ so $E(R / J) \cdot\left(\bigcap_{i=1}^{n} D_{i}\right) J=0$. We conclude $\left(\bigcap_{i=1}^{n} D_{i}\right) J=0$, so $\bigcap_{i=1}^{n} D_{i} \subseteq^{\prime} S(R) \subseteq \subseteq^{\prime} R$.

We now come to a basic lemma on restricted hypercyclic rings, extending a result in Faith [3], who proved it in the case that $R$ 
was perfect and hypercyclic.

LeMмA 1.7. Let $R$ be a hypercyclic ring such that every homomorphic image of $R$ has ascending chain condition on direct summands. Then $R_{R}$ is injective and $R$ is restricted hypercyclic.

Proof. Since $R$ is hypercyclic, $E(R) \approx R / I$ for some right ideal $I$. Let $f$ embed $R$ in $R / I, f(1)=x+I$. Since $f$ is one-to-one, $x R \cap I=$ 0 , so $E(R)=E(x R) \oplus E(I) \oplus M$. Since $x R \approx R, E(x R) \approx E(R) \oplus E(I) \oplus$ $M \quad$ so $\quad E(R) \approx E(R) \oplus E(I) \oplus M \oplus E(I) \oplus M \approx E(R) \oplus E(I) \oplus M \oplus$ $E(I) \oplus M \oplus E(I) \oplus M \approx \cdots \approx E(R) \oplus \sum_{i=1}^{n}[E(I) \oplus M]_{i}$. Since $R / I$ has ascending chain condition on direct summands, $E(I) \oplus M=0$, so $I=0$ and $R_{R}$ is injective. Since $R / J$ is regular by 1.3 , the chain condition on $R / J$ implies $R / J$ artinian.

Lemma 1.8. Let $R / J$ be artinian, $I$ a right ideal of $R, R / I=$ $\sum_{i=1}^{m} \oplus M_{i}$. Then $m \leqq$ the composition length of $R / J$. Moreover, if $M_{i} / M_{i} J$ is simple for each $i$, the number of $M_{i} / M_{i} J$ isomorphic to a given simple module $M \leqq$ the number of factor modules $\approx M$ in a composition series for $R / J$.

Proof. $\quad \sum_{i=1}^{m} \oplus M_{i} / M_{i} J \approx(R / I) /(R / I) J \approx R / I+J \approx(R / J) /(I+J / J)$. The lemma then follows from the Jordan-Holder-Schreier theorem. See Jacobson [7], p. 141.

COROLlARY 1.9. Let $R$ be restricted hypercyclic, $e=e^{2} \in R$, length $e R / e J=m$. Then any independent set of submodules of a quotient of $\mathrm{e} R$ has at most $m$ elements.

Proof. Let $\left\{M_{i} \mid 1 \leqq i \leqq k\right\}$ be an independent family of submodules of $e R / e I$. Then $R / e I \supseteqq(1-e) R \oplus \sum_{i=1}^{k} \oplus M_{i}$, so $E(R / e I)$ contains a direct sum of (length $R / J-m+k$ ) terms. Hence $k \leqq m$ by 1.8 .

COROLLARY 1.10. Let $R$ be restricted hypercyclic, $e=e^{2} \in R$, $e R / e J$ simple. Then the submodules of eR are linearly ordered.

Proof. Let $A, B \subseteq e R$. By $1.9, A / A \cap B=0$ or $B / A \cap B=0$, so $B \subseteq A \cap B$ or $B \subseteq A \cap B$.

CoRollaRY 1.11. Let $R$ be restricted hypercyclic. Then $R_{R}$ is injective.

Proof. Apply 1.8 and 1.7. 
LEMMA 1.12. Let $R$ be restricted hypercyclic, $M$ a simple $R$ module. Then $E(M) \approx e R$ for some $e=e^{2} \in R$.

Proof. By 1.11, $R_{R}$ is injective. Let $R / J=\sum_{i=1}^{m} \varepsilon_{i} R,\left\{\varepsilon_{i} \mid 1 \leqq\right.$ $i \leqq m\}$ orthogonal primitive idempotents. By 1.3 , there exist $\left\{e_{i} \mid 1 \leqq\right.$ $i \leqq m\}$ orthogonal idempotents of $R$ such that $e_{i}+J=\varepsilon_{i}$ and $1=$ $\sum_{i=1}^{m} e_{i}$. By 1.6, $e_{i} R \cap S(R) \neq 0$. Since $e_{i} R / e_{i} J$ is indecomposable, so is $e_{i} R=E\left(e_{i} R \cap S(R)\right)$. Hence $e_{i} R \cap S(R)=S_{i}$ is simple. By 1.4, $e_{i} R / e_{i} J \approx e_{j} R / e_{j} J \Leftrightarrow S_{i} \approx S_{j}$. Hence the correspondence $S_{i} \leftrightarrow E\left(S_{i}\right) / E\left(S_{i}\right) J$ is one-to-one between isomorphism classes of simple modules containing a representative $S_{i} \subseteq S(R)$ and isomorphism classes of modules of the form $e_{i} R / e_{i} J$. Since every simple $R$-module is isomorphic to some $e_{i} R / e_{i} J$ and the set of isomorphism classes of simples is finite, every isomorphism class of simples contains some $S_{i}$. If $M \approx S_{i_{0}}, E(M) \approx$ $e_{i_{0}} R$.

Lemma 1.13. Let $R$ be hypercyclic, $R / J \approx \Delta_{n}$ for some division ring 4 . Then $R \approx\left(R^{\prime}\right)_{n}$, where $R^{\prime} / R^{\prime} J\left(R^{\prime}\right) \approx \Delta$, so $R^{\prime}$ is local.

Proof. Let $R / J=\sum_{i=1}^{m} \varepsilon_{i} R / J,\left\{\varepsilon_{i} \mid 1 \leqq i \leqq m\right\}$ primitive orthogonal idempotents. Then $R=\sum_{i=1}^{m} e_{i} R,\left\{e_{i} \mid 1 \leqq i \leqq m\right\}$ orthogonal idempotents with $e_{i}+J=\varepsilon_{i}$ by 1.11 and 1.3. By 1.4, $e_{i} R \approx e_{j} R$ for all $i$ and $j$ since all simple $\Delta_{n}$ modules are isomorphic. By 1.2, $R \approx\left(\operatorname{Hom}_{R}\left(e_{i} R\right.\right.$, $\left.\left.e_{i} R\right)\right)_{n}$. But $R^{\prime}=\operatorname{Hom}_{R}\left(e_{i} R, e_{i} R\right) \approx e_{i} R e_{i}$, and $R^{\prime} / R^{\prime} J\left(R^{\prime}\right) \approx e_{i} R e_{i} / e_{i} J e_{i} \approx$ $\operatorname{Hom}_{R / J}\left(\varepsilon_{i} \Delta_{n}, \varepsilon_{i} \Delta_{n}\right) \approx \Delta$.

Lemma 1.14. Let $R=\sum_{i=1}^{n} \oplus R_{i}$ be a ring direct sum. Then $R$ is hypercyclic $\Leftrightarrow$ each $R_{i}$ is.

Proof. If $e_{i}$ is the identity of $R_{i}$, then $\left\{e_{i} \mid 1 \leqq i \leqq n\right\}$ are orthogonal central idempotents of $R$. Let $I$ be an ideal of $R$. Then $I=\sum \oplus e_{i} I$, and one easily verifies that $E(R / I) \approx R / K \Leftrightarrow E\left(e_{i} R / e_{i} I\right) \approx$ $e_{i} R / e_{i} K$.

Lemma 1.15. Let $R$ be restricted hypercyclic. Then $R$ is a ring direct sum of matrix rings over local rings.

Proof. Let $R / J=\sum_{i=1}^{m} \oplus\left(\Delta_{i}\right)_{n_{i}}, \Delta_{i}$ a division ring, $n_{1} \geqq n_{2} \geqq \cdots \geqq$ $n_{m},\left(\Delta_{i}\right)_{n_{i}}=\varepsilon_{i} R / J$ where $\varepsilon_{i}$ is a central idempotent of $R / J$. Let $\varepsilon_{i}=$ $e_{i}+J,\left\{e_{i} \mid 1 \leqq i \leqq m\right\}$ orthogonal idempotents of $R$ such that $\sum_{i=1}^{m} e_{\imath}=$ 1. By Lemmas 1.13 and 1.14, we need only show each $e_{i}$ is central. Assume not. Then there exist $i \neq j$ such that $e_{i} R e_{j} \neq 0$. Let $e_{i} r e_{j} \neq$ $0, i \neq j$. Consider 


$$
M=R /\left(\sum_{l \neq i, j} e_{l} R+e_{j} J+e_{i} r e_{j} J\right) \approx e_{j} R / e_{j} J \oplus e_{i} R / e_{i} r e_{j} J .
$$

This contains a direct sum of at least $n_{j}+1$ copies of the unique simple $\left(\Delta_{j}\right)_{n_{j}}$ module $S$. Then $E(M) \supseteqq \sum_{i=1}^{n_{j}+1} E(S) \approx R / I$ for some $I$. Since $E(S) / E(S) J$ is simple by the proof of 1.12 , lemma 1.8 shows $E(S) / E(S) J$ is the simple $\left(\Delta_{l}\right)_{n_{l}}$ module for some $l$ with $n_{l}>n_{j}$. Now $E(R / J) \approx R / K$ for some $K$, and by 1.12 , since $R / J$ is a finite direct sum of simple modules, $E(R / J)$ is projective. Hence $R \approx E(R / J) \oplus K$, where the length of $E(R / J) / E(R / J) J=$ length $R / J$. By 1.8, $K=0$, so $S(R) \approx R / J$. But then the number of composition factors of $S(R) \approx$ $S=$ the number of times $E(S) / E(S) J$ appears as a composition factor of $R / J=$ the number of composition factors of $R / J \approx S$. Thus $n_{l}=$ $n_{j}$, a contradiction.

Lemmas 1.14 and 1.15 reduce the study of restricted hypercyclic rings to hypercyclic matrix rings over local rings $R^{\prime}$. We will show that, for $R^{\prime}$ local, $\left(R^{\prime}\right)_{n}$ is hypercyclic $\Leftrightarrow R^{\prime}$ is hypercyclic.

Lemma 1.16. Let $R$ be hypercyclic, $R / J$ a simple $R$-module. Let $F=\sum_{1}^{n} \oplus R_{i}, K \subseteq F$. Let $\left\{N_{i} \mid 1 \leqq i \leqq k\right\}$ be a family of nonzero independent submodules of $F / K$. Then $k \leqq n$.

Proof. Let $x_{1}, \cdots, x_{n+1}$ be any $n+1$ elements of $F, x_{i}=\left(x_{i 1}, \cdots\right.$, $\left.x_{i n}\right)$. We will show that $\left\{x_{i} \mid 1 \leqq i \leqq n+1\right\}$ is not independent modulo $K$. If some $x_{i} \in K$, this is immediate, so we may assume $x_{i} \notin K$ for all $i$. By 1.10, the right ideals of $R$ are linearly ordered. Hence any finite subset of $R,\left\{r_{i}\right\}$, has a maximum element, that is an $r_{i_{0}}$ such that $r_{i_{0}} R \supseteqq r_{i} R$ for all $i$. Clearly we may permute the $x_{i}$ and the order of the summands $R_{i}$ in $F$ without losing generality. Hence we may assume $x_{11}=\max \left\{x_{i j} \mid 1 \leqq i \leqq n+1,1 \leqq j \leqq n\right\}$. Let $x_{i 1}=$ $x_{11} r_{1 i}, 2 \leqq i \leqq n+1$, and consider the elements $x_{i}-x_{1} r_{1 i}$. These all have zeros in the first component. Then some $x_{i j}-x_{1 j} r_{1 i}=\max \left\{x_{i j}\right.$ $\left.x_{1 j} r_{1 i} \mid 2 \leqq i \leqq n+1,2 \leqq j \leqq n\right\}$, say $i=j=2$. Then there exist elements $r_{2 i}, 3 \leqq i \leqq n+1$ such that $\left\{\left(x_{i}-x_{1} r_{1 i}\right)-\left(x_{2}-x_{1} r_{12}\right) r_{2 i} \mid 3 \leqq\right.$ $i \leqq n+1\}$ all have zeros in the first two components. Continuing in this manner, and permuting so the largest coefficient is in the $k, k$ position, we get $n+1-k$ elements of the form $x_{m}-\sum_{i=1}^{k} x_{i} s_{i k}$ which have zeros in the first $k$ positions. When $k=n$, we get $x_{n+1}$ $\sum_{i=1}^{n} x_{i} s_{i n}=0$, and since $x_{n+1} \notin K$, this gives a nontrivial dependence of $\left\{x_{i} \mid 1 \leqq i \leqq n+1\right\}$ modulo $K$.

Lemma 1.17. Let $R^{\prime}$ be a local ring. Then $R^{\prime}$ is hypercyclic $\Leftrightarrow\left(R^{\prime}\right)_{n}$ is hypercyclic for some $n$. 
Proof. $\Rightarrow$ Let $R^{\prime}$ be hypercyclic, $e=e^{2} \in\left(R^{\prime}\right)_{n}=R, e$ a primitive idempotent. Identify $R^{\prime}$ with $e R e$. Then $R e_{R^{\prime}} \approx \sum_{i=1}^{n} \oplus R_{i}^{\prime}$. Since the category isomorphism of 1.5 takes $R^{\prime} \rightarrow \operatorname{Hom}_{R^{\prime}}\left(R e, R^{\prime}\right) \approx e R$, every quotient of $e R$ has injective hull a quotient of $e R$. Let $I$ be a right ideal of $R$. Then

$$
R \rightarrow R / I \rightarrow 0
$$

is exact, so

$$
R \otimes_{R} R e \rightarrow R / I \otimes_{R} R e \rightarrow 0
$$

is exact. Since $R \otimes_{R} R e_{R^{\prime}} \approx R e_{R^{\prime}}, R / I \otimes_{R} R e$ is an $R^{\prime}$-quotient of $R e$. By 1.16, $R / I \otimes_{R} R e$ is an essential extension of at most $n$ cyclic $R^{\prime}$ modules. By the category isomorphism, $R / I$ is an essential extension of a sum of at most $n$ quotients of $e R$. Hence its injective hull is a direct sum of at most $n$ quotients of $e R$, and thus a quotient of $R$. $\Leftarrow$. Let $R=\left(R^{\prime}\right)_{n}$ be hypercyclic, $e$ a primitive idempotent, $A \subseteq$ $e\left(R^{\prime}\right)_{n}$. Then $E(R / A)=(1-e) R \oplus E(e R / A)$, so $E(e R / A) / E(e R / A) J$ is simple, and $E(e R / A)$ is a quotient of $e R$. By the category isomorphism 1.5, every quotient of $R^{\prime}$ has injective hull also a quotient of $R^{\prime}$.

Putting 1.17, 1.15 and 1.14 together we get

THEOREM 1.18. $R$ is a restricted hypercyclic ring $\Leftrightarrow R$ is a ring direct sum of matrix rings over local hypercyclic rings.

2. Local hypercyclic rings. By Theorem 1.18, hypercyclic rings $R$ such that $R / J$ is semi-simple Artin are ring direct sums of matrix rings over local hypercyclic rings. In this section we study local hypercyclic rings. These turn out to be, with one possible exception, the rings studied by Caldwell in [2], with $R$ commutative replaced by $x R=R x$ for all $x \in R$.

By 1.11, a local hypercyclic ring is right self injective; by 1.10 its right ideals are linearly ordered.

We have the well-known

Proposition 2.1. Let $M_{R}$ contain a copy of the injective hull of every simple $R$-module. Then every right ideal of $R$ is the annihilator of some subset of $M$.

Proposition 2.2. Let $R_{R}$ be injective. Then any finitely generated left ideal is the annihilator of some right ideal, and the right socle of $R \cong$ the left socle of $R$.

Let $X \subseteq R$. Define $X^{r}=\{r \in R \mid X r=0\}, X^{\imath}=\{r \in R \mid r X=0\}$. 
CoRollary 2.3. Let $R$ be a local hypercyclic ring. Then the left ideals of $R$ are linearly ordered.

Proof. Let $x_{1}, x_{2} \in R$. Then $x_{1}^{r} \subseteq x_{2}^{r}$ or $x_{2}^{r} \subseteq x_{1}^{r}$, so by $2.2, x_{1}^{r l}=$ $R x_{1} \subseteq x_{2}^{r l}=R x_{2}$ or $R x_{1} \supseteqq R x_{2}$. Now let $I_{1}, I_{2}$ be two left ideals, $I_{1} \nsubseteq I_{2}$. Let $x \in I_{1}-I_{2}$. For $y \in I_{2}, R y \nsupseteq R x$ since $x \notin I_{2}$. Hence $R y \subseteq R x$ so $I_{2} \cong I_{1}$.

Lemma 2.4. Let $x, z \in R, 0 \neq x z \in S(R) . \quad$ Then $(R x)^{r}=z J,(J x)^{r}=$ $z R,(z J)^{l}=R x,(z R)^{l}=J x$.

Proof. Since $0 \neq x z \in S, x z J=0, x z R \neq 0$. By the linear ordering on right ideals, $z J \subseteq(R x)^{r} \varsubsetneqq z R$. Since $z R / z J$ is simple, $z J=(R x)^{r}$. Since left ideals are linearly ordered by $2.3, J x=(z R)^{l}$ by symmetry. By 2.2, principal left ideals are annihilators so $R x=(z J)^{l}$. By 2.1, $z R$ is an annihilator so $(J x)^{r}=z R$.

We note that given $x \neq 0$ or $z \neq 0$, by the linear ordering on right and left ideals, we can always find the other such that $0 \neq$ $x z \in S(R)$.

COROLlaRY 2.5. Let $R$ be a local hypercyclic ring. Then every right ideal and every left ideal is an annihilator ideal.

Proof. Since $R_{R}$ is injective and contains a copy of the unique simple $R$-module, 2.1 states every right ideal is a right annihilator.

Now let $I^{\prime}$ be a left ideal, and let $Z=\bigcap_{R x \geqq I^{\prime}} R x$. Then $Z=$ $\left(\sum_{R x \supseteq I^{\prime}} x^{r}\right)^{l}$ since $\left(x^{r}\right)^{l}=R x$. If $I^{\prime} \neq Z$, let $y \in Z-I^{\prime}$. Then $R y \supseteqq I^{\prime}$, and $R z \supseteqq I^{\prime} \Rightarrow R z \supseteqq R y$. Hence $R y / I^{\prime}$ is a simple left module. By Nakayama's lemma, $J y \neq R y$. Hence $J y \cong I^{\prime}$. Since $R y / J y$ is also simple, $J y=I^{\prime}$. By 2.4, $I^{\prime}$ is an annihilator left ideal.

LEMMA 2.6. Let $I$ be a right ideal of the local hypercyclic ring $R, R \supsetneqq I \supsetneqq S(R)$. Then $R / I$ is not injective.

Proof. Since $I \neq R, I \subseteq J$. Let $x \in I, x \notin S(R)$. Since the left ideals of $R$ are linearly ordered and $S(R)$ is a two sided ideal, there exists $y \in R$ such that $0 \neq y x \in S(R)$. Then $x J=(R y)^{r}$ by 2.4. Since $x \notin S(R), y$ is not a unit, so $y \in J$. We now proceed as in Caldwell [2]; the map $f: y R \rightarrow R / I$ given by $f(y r)=r+I$ is well defined since $y^{r}=x J \subseteq I . \quad R / I$ injective implies there exists $m \in R$ such that $m y+$ $I=1+I$. Hence $1-m y \in I$, and since $y \in J,(1-m y)(1-m y)^{-1}=$ $1 \in I$, a contradiction.

LEMMA 2.7. Let $R$ be local hypercyclic. Then every right (left) 
ideal is of the form $x R$ or $x J(R x$ or $J x)$.

Proof. Let $I$ be a right ideal. Since the injective hull of $R / I$ is cyclic, by $2.6 R / I$ embeds either in $R$ or in $R / S(R)$. Assume $f$ embeds $R / I$ in $R$. Let $0 \neq f(x+I) \in S(R)$. Then $x J \cong I \varsubsetneqq x R$, so $I=x J$. If $g$ embeds $R / I$ in $R / S(R)$, let $g(1)=m+S(R)$. Let $0 \neq$ $m y \in S(R)$. Then $g(y)=0$ so $y \in I$. Let $x \in I$. Then $m x \in S(R)$. If $m x=0, x \in y J=m^{r}$. If $m x \neq 0, m^{r}=x J=y J$, so by the linear ordering $x R=y R$. Hence $x \in y^{R}$, and $I=y R$.

Since every left ideal $I$ is an annihilator by 2.5 , it is of the form $(x R)^{l}$ or $(x J)^{l}$ for some $x$. If $x \neq 0$, select $y$ such that $0 \neq y x \in S(R)$. Then $I=J y$ or $R y$ by 2.4 .

Proposition 2.8. Let $R$ be local hypercyclic, $y \in J$. Either $y$ is nilpotent or $0 \neq \bigcap_{i=0}^{\infty} y^{n} R=z^{\prime} R$, where $y z^{\prime} R=z^{\prime} R$.

Proof. (See Caldwell [2] Theorem 2.20) Since $R^{\prime} \supseteqq S(R), y^{n} R \supseteqq S(R)$ or $y^{n}=0$. Thus $I=\bigcap_{n=0}^{\infty} y^{n} R=0 \Leftrightarrow y^{n}=0$ for some $n$.

Let $y^{n} \in y^{n+1} R$. Then for some $r \in R, y^{n}=y^{n+1} r$, so $y^{n}(1-y r)=$ 0 , and since $y r \in J, 1-y r$ is invertible. Hence $y^{n}=0$. Thus $y$ not nilpotent implies $y R \supset y^{2} R \supset \cdots$ is a strictly descending chain of right ideals. Let $\left(y^{i} R\right)^{l}=J z_{i}$. Then $I=\left(\bigcup_{i=0}^{\infty} J z_{i}\right)^{r}$, where $\bigcup_{i=0}^{\infty} J z_{i}=K$ is the union of a strictly ascending chain of left ideals if $y$ is not nilpotent. Then $K$ cannot be finitely generated so $K=J z$ for some $z \in R$ by 2.7 , and $I=K^{r}=z^{\prime} R$ for some $z^{\prime}$. Since $y\left(y^{n} R\right) \subseteq y^{n} R$ for all $n, y I \subseteq I$. Now $z^{\prime} \in I$, so $z^{\prime}=y r$ for some $r \in R$. Assume $r \notin I$. Then there is an $n$ such that $r \notin y^{n} R$, so $r R \supset y^{n} R$. Let $r s=y^{n}$. Then $z^{\prime} s=y r s=y^{n+1} \in I$. If $y$ is not nilpotent, this cannot occur, so $r \in I$ and $z^{\prime} \in y I$. Hence $y I=I$.

CoRollaRy 2.9. Let $y R=R y$ for $y \in J$. Then $y$ is nilpotent.

Proof. Assume not. Then $0 \neq I=\bigcap_{i=0}^{\infty} y^{n} R=\bigcap_{i=0}^{\infty} R y^{n}$. As in the proof of 2.8, $\left(\bigcap_{i=0}^{\infty} R y^{n}\right) y=\bigcap_{i=0}^{\infty} R y^{n}$. By 2.8, for some $z^{\prime}, I=$ $z^{\prime} R$. Then $I=z^{\prime} R y$, so there exists $r \in R$ with $z^{\prime} r y=z^{\prime}, z^{\prime}(r y-1)=$ 0 . Since $y \in J, r y-1$ is invertible, so $z^{\prime}=0$, a contradiction.

THEOREM 2.10. Let $R$ be local hypercyclic. Then $J$ is nil $\Leftrightarrow y R=$ $R y$ for all $y \in R$.

Proof. $\Longleftarrow$. This is just 2.9. $\Rightarrow$. Assume $J$ is nil, and let $0 \neq$ $y, r \in R, y r \notin R y$. Then $R y r \supsetneqq R y$, so $y=x y r$ for some $x \in J$. Then $y=x y r=x^{2} y r^{2}=\cdots=x^{n} y r^{n}=0$, a contradiction. Hence $y R \cong R y$. By symmetry, $R y \subseteq y R$.

Thus if $J$ is nil, every one sided ideal of $R$ is two sided, and all 
of Caldwell's arguments in [2] may be carried over to this case almost verbatim. The reader is referred to Caldwell for further discussion of this case.

Whether a local hypercyclic ring can have nonnil radical is unknown. We show that it implies the existence of a very elusive type of ring, namely a simple radical ring (without 1 of course), which has linear ordering on both right and left ideals.

LemMA 2.11. Let $R$ be a hypercyclic local ring, $J$ not nil. Then $J^{2}=J$ and for all $y \in J, J y J \neq J \Rightarrow \exists z \in J, z R=R z$ and $y \in z R$.

Proof. If $J^{2} \neq J$, by the linear ordering on one sided ideals, for any $x \in J-J^{2}, J=x R=R x$. Hence $x$ is nilpotent by 2.9 , so $J^{n}=$ $(x R)^{n}=x^{n} R=0$ for some $n$, a contradiction. Hence $J=J^{2}$. Let $y \in J$. Then $(J y J) J=J y J=J(J y J)$, so $J y J$ is not a finitely generated right or left ideal. Hence $J y J=z J=J z^{\prime}$ for some $z, z^{\prime} \in R$ by 2.7. Let $\nu$ denote the natural map from $R$ onto $R / J y J$. Then $\nu(z) R=$ $S\left(\nu(R)_{R}\right), R \nu\left(z^{\prime}\right)=S\left(_{R} \nu(R)\right)$ since the right and left ideals of $\nu(R)$ are linearly ordered and $\nu(z) R$ and $R \nu\left(z^{\prime}\right)$ are simple. Moreover, $\nu\left(z^{\prime}\right) R \supseteqq$ $\nu(z) R$. Since $S\left({ }_{R} \nu(R)\right)$ is a two sided ideal of $\nu(R), S\left({ }_{R} \nu(R)\right)=R \nu\left(z^{\prime}\right) \subseteq$ $\nu(z) R=S\left(\nu(R)_{R}\right)$. By symmetry, $S\left(\nu(R)_{R}\right) \subseteq S\left({ }_{R} \nu(R)\right)$, so $R \nu\left(z^{\prime}\right)=$ $\nu(z) R=\nu\left(z^{\prime}\right) R=R \nu(z)$ since it is a simple $R$-module on both right and left. Taking $\nu^{-1}$ of both sides we get $z^{\prime} R=R z^{\prime}=z R=R z$. If $J y J \neq J, S\left(\nu(R)_{R}\right) \neq R / J$, so $z \in J$.

Moreover $z R \supsetneq J y J \Longrightarrow z R \supsetneq j y J$ for all $j \in J \Rightarrow z R \supseteqq j y R$ for all $j \in J$ by the linear ordering $\Rightarrow z R \supseteqq J y R$. Similarly $z R=R z \supseteqq R y R$. Hence $y \in z R$.

THEOREM 2.12. Let $R$ be a local hypercyclic ring, $J$ not nil. Then there exists a nilpotent ideal $z R \subseteq J$ such that $z R$ is a maximal proper two sided ideal of $J$ (so $J / z R$ is a simple radical ring.)

Proof. Let $I$ be the union of all the nil two-sided ideals of $R$. Then $I$ is a nil two-sided ideal since ideals are linearly ordered. Moreover, $I \neq 0$ since $S$ is a nilpotent two-sided ideal, and $I \neq J$ since $J$ is not nil.

Let $K=J I J$. Then $J K=K J=K$, so as in $2.11, K=z J=J z$ where $z R=R z=\nu^{-1}(S(R / K))$ and $x \in R z$ for all $x \in I$. Since

$$
\left[\nu^{-1}(S(R / K))\right]^{2} \subseteq K
$$

and $K \subseteq I, z R$ is nil. Hence $z R=R z=I$. If $z^{n}=0$, then $I^{n}=$ $(z R)^{n}=z^{n} R=0$, so $I$ is nilpotent.

Now let $y \in J-I$. If $J y J \neq J$, by $2.11, y$ belongs to some nilpotent ideal of $R$ and hence to $I$. Thus $J y J=J$, and $J / z R$ is a 
simple ring. By [8], p. $10, J$ is a radical ring, and hence so is $J / z R$.

\section{REFERENCES}

1. W. Caldwell, Rings for which cyclic modules have cyclic injective hulls, Ph. D. thesis, Rutgers, The State University, 1966.

2. - Hypercyclic rings, Pacific J. Math. 24 (1968), 29-44.

3. C. Faith, On Köthe rings, Math. Ann. 164 (1966), 207-212.

4. L Lectures on Injective Modules and Quotient Rings, Springer Verlag, New York, 1967.

5. C. Faith and Y. Utumi, Quasi-injective modules and their endomorphism rings, Arch. Math. 15 (1964), 166-174.

6. N. Jacobson, The radical and semi-simplicity for arbitrary rings, Amer. J. Math. 67 (1945), 300-320.

7. Lectures in Abstract Algebra, Volume 1, Van Nostrand, Princeton, 1951.

8. - Structure of Rings, Amer. Math. Soc. Colloquium, Vol. 36, Providence,

R. I., 1964.

9. J. P. Jans, Rings and Homology, Holt, Rinehart, and Winston, New York, 1964.

10. K. Morita, Duality for modules and its applications to the theory of rings with minimum condition, Tokyo, Kyoiku Daigaku, Section A, 6, (1958), 83-142.

11. T. Nakayama, A remark on finitely generated modules, Nagoya Math. J. 3 (1951), 139-140.

12. B. Osofsky, A generalization of quasi-Frobenius rings, Journal of Algebra 4 (1966), 373-387.

Received January 18, 1967. The author gratefully acknowledges partial support from the National Science Foundation under grant GP-4226.

Rutgers, The State University 


\section{PACIFIC JOURNAL OF MATHEMATICS}

\section{EDITORS}

\section{H. ROYDEN}

Stanford University

Stanford, California
J. DugundJI

Department of Mathematics

Rice University

Houston, Texas 77001

RICHARD ARENS

University of California

Los Angeles, California 90024

Seattle, Washington 98105

\section{ASSOCIATE EDITORS}
E. F. BeCKENBACH
B. H. NeumanN
F. WOLF
K. YOSIDA

\section{SUPPORTING INSTITUTIONS}

\author{
UNIVERSITY OF BRITISH COLUMBIA \\ CALIFORNIA INSTITUTE OF TECHNOLOGY \\ UNIVERSITY OF CALIFORNIA \\ MONTANA STATE UNIVERSITY \\ UNIVERSITY OF NEVADA \\ NEW MEXICO STATE UNIVERSITY \\ OREGON STATE UNIVERSITY \\ UNIVERSITY OF OREGON \\ OSAKA UNIVERSITY \\ UNIVERSITY OF SOUTHERN CALIFORNIA
}

\author{
STANFORD UNIVERSITY \\ UNIVERSITY OF TOKYO \\ UNIVERSITY OF UTAH \\ WASHINGTON STATE UNIVERSITY \\ UNIVERSITY OF WASHINGTON \\ * * * * \\ AMERICAN MATHEMATICAL SOCIETY \\ CHEVRON RESEARCH CORPORATION \\ TRW SYSTEMS \\ NAVAL WEAPONS CENTER
}

Mathematical papers intended for publication in the Pacific Journal of Mathematics should be in typed form or offset-reproduced, double spaced with large margins. Underline Greek letters in red, German in green, and script in blue. The first paragraph or two must be capable of being used separately as a synopsis of the entire paper. It should not contain references to the bibliography. Manuscripts, in duplicate if possible, may be sent to any one of the four editors. All other communications to the editors should be addressed to the managing editor, Richard Arens, University of California, Los Angeles, California 90024.

Each author of each article receives 50 reprints free of charge; additional copies may be obtained at cost in multiples of 50 .

The Pacific Journal of Mathematics is published monthly. Effective with Volume 16 the price per volume (3 numbers) is $\$ 8.00$; single issues, $\$ 3.00$. Special price for current issues to individual faculty members of supporting institutions and to individual members of the American Mathematical Society: $\$ 4.00$ per volume; single issues $\$ 1.50$. Back numbers are available.

Subscriptions, orders for back numbers, and changes of address should be sent to Pacific Journal of Mathematics, 103 Highland Boulevard, Berkeley 8, California.

Printed at Kokusai Bunken Insatsusha (International Academic Printing Co., Ltd.), 7-17, Fujimi 2-chome, Chiyoda-ku, Tokyo, Japan.

PUBLISHED BY PACIFIC JOURNAL OF MATHEMATICS, A NON-PROFIT CORPORATION

The Supporting Institutions listed above contribute to the cost of publication of this Journal, but they are not owners of publishers and have no responsibility for its content or policies. 


\section{Pacific Journal of Mathematics \\ Vol. 25, No. $2 \quad$ October, 1968}

Martin Aigner, On the tetrahedral graph ..................... 219

Gregory Frank Bachelis, Homomorphisms of annihilator Banach

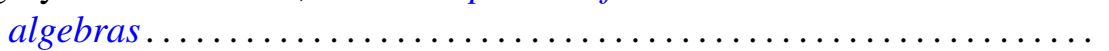

Phillip Alan Griffith, Transitive and fully transitive primary abelian groups.......................................... 249

Benjamin Rigler Halpern, Fixed points for iterates . . . . . . . . . . . . . 255

James Edgar Keesling, Mappings and dimension in general metric spaces ......................................... 277

$\mathrm{Al}$ (Allen Frederick) Kelley, Jr., Invariance for linear systems of ordinary differential equations ................................ 289

Hayri Korezlioglu, Reproducing kernels in separable Hilbert spaces . . . . . 305

Gerson Louis Levin and Wolmer Vasconcelos, Homological dimensions and Macaulay rings ................................. 315

Leo Sario and Mitsuru Nakai, Point norms in the construction of harmonic



Barbara Osofsky, Noncommutative rings whose cyclic modules have cyclic injective hulls ..................................... 331

Newton Tenney Peck, Extreme points and dimension theory........... 341

Jack Segal, Quasi dimension type. II. Types in 1-dimensional spaces ...... 353

Michael Schilder, Expected values of functionals with respect to the Ito distribution ...

Grigorios Tsagas, A Riemannian space with strictly positive sectional curvature

John Alexander Williamson, Random walks and Riesz kernels . . 